\title{
Vitamin C protects against chronic social isolation stress-induced weight gain and depressive-like behavior in adult male rats
}

\author{
Alireza Najaf Dulabi ${ }^{1}$, Zeinab Shakerin ${ }^{2}$, Nasrin Mehranfard ${ }^{3}$, Maedeh Ghasemi ${ }^{4}$ \\ ${ }^{1}$ School of Medicine, Isfahan University of Medical Sciences, Isfahan, Iran; ${ }^{2}$ Department of Anatomy, School of Medi- \\ cine, Isfahan University of Medical Sciences, Isfahan, Iran; ${ }^{3}$ Neurophysiology Research Center, Cellular and Molecular \\ Medicine Institute, Urmia University of Medical Sciences, Urmia, Iran; ${ }^{4}$ Department of Physiology, School of Medicine, \\ Isfahan University of Medical Sciences, Isfahan, Iran \\ E-mail: ghasemi.m@med.mui.ac.ir
}

\begin{abstract}
Objective. Considering the importance of ghrelin in stress-induced hyperphagia and a role of antioxidants in decreasing body weight, in the present study, the effect of vitamin C (VitC) on ghrelin secretion and food intake following chronic social isolation (CIS) was evaluated in rats.

Methods. Thirty two male Wistar rats (200-220g) were randomly divided into: control, VitC, CIS, and CIS + VitC groups. Animals received VitC ( $500 \mathrm{mg} / \mathrm{kg} /$ day $) /$ saline by gavage for 3 weeks. For $24 \mathrm{~h}$ cumulative and post $18-20 \mathrm{~h}$ fasting food intake, fasting plasma ghrelin level, and body weight were measured. Gastric histopathology was also evaluated.

Results. Results showed a marked increase in fasting plasma ghrelin and food intake in stressed rats compared to controls. VitC prevented the increases in stressed rats. Histological assessment indicated a positive effect of VitC on gastric glandular cells compared to control, an effect that might partially be a reason of significant increase of plasma ghrelin levels in VitC rats. Elevated plasma ghrelin in VitC group was even higher than that one in stressed group, whereas there were no significant changes in the food intake. Assessment of the percentage of changes in body weight during 21 days showed a significant increase in stressed rats compared to controls. Vitamin C treatment prevented this increase. Stressed rats also displayed depression-like behavior as indicated by sucrose test, whereas VitC ameliorated it.

Conclusions. The data of the present study indicate that VitC may overcome ghrelin-induced hyperphagia and improve the abnormal feeding and depressive behavior in CIS rats.
\end{abstract}

Key words: ghrelin, vitamin C, food intake, chronic isolation stress, depression-like behavior

The chronic isolation stress (CIS) is implicated in persistent hyperphagia and binge eating disorders (Teegarden and Bale 2008; Razzoli et al. 2015). Several previous studies have demonstrated a principal role of the gut-derived hormone ghrelin in the central responses to stress, mood, and food intake pattern primarily via modulation of the orexigenic pathways in hypothalamus (Muller et al. 2015). In animal models of chronic stress, the plasma concentration of acyl-ghrelin is increased (Cummings et al. 2002;
Kristenssson et al. 2006; Ouach et al. 2007; Lutter et al. 2008a, b; Ochi et al. 2008; Chuang and Zigman 2010; Chuang 2011; Patterson et al. 2013) and persists beyond the cessation of stressor exposure (Lutter et al. 2008a; Kumar et al. 2013). Ghrelin is found to mediate chronic stress-induced increase in hyperphagia and weight gain and genetic or pharmacological blockade of its receptor (growth hormone secretogauge receptor; GHSR) have been shown to suppress this effect (Chuang 2011; Patterson et al. 2013). Although, 
the hyperphagic effect of ghrelin is known to maintain energy homeostasis under stress condition, it also can increase production of reactive oxidative species (Bisbal et al. 2010). Clinical reports have indicated close interactions between stressors, particularly those of long duration, and incidence of different diseases that occur mainly via cellular oxidative stress (Pizzino et al. 2017). Hence, antioxidant supplements may be useful in the management of some diseases related to oxidative stress (Valdecantos et al. 2009; Abdali et al. 2015). It has been reported that obese patients often have significant reductions in vitamins level, in particular vitamins A, B6, C, D, and E (Aasheim and Bohmer 2009). Deficiencies in vitamins and minerals have been associated with impaired antioxidant defense in the pathogenesis of obesity (Strauss 1999; Ortega et al. 2012). Due to the existence of correlation between obesity and its major associated disorders, a potential beneficial effect of antioxidant supplements in decreasing obesity and several obesity-associated disorders has been suggested by previous studies (Valdecantos et al. 2009; Abdali et al. 2015). Therefore, considering the importance of ghrelin in stressinduced hyperphagia as well as due to a powerful antioxidant effect of vitamin $\mathrm{C}(\mathrm{Vit} C)$, the aim of the present study was to examine the antioxidant impact of VitC on ghrelin secretion and its orexigenic effects following CIS in adult male rats.

\section{Material and methods}

Drug administration. Acid ascorbic was purchased from Osvah Chemical Co. (Iran). Acid ascorbic solution $(500 \mathrm{mg} / \mathrm{kg}$; freshly suspended in saline) (Beheshti et al. 2017) was administrated by gavage once a day for 3 weeks.

Ethical considerations. Animal proceedings were conducted in accordance with the international guiding principles for biomedical research involving animals in 1996 (NIH Publications No. 80-23, 1996 Rev) and were approved by the Research and Ethics Committee of Isfahan University of Medical Science (IR.MUI.MED.REC.1397.142).

Animals and treatment. Thirty-two male Wistar rats (200-220 g) were obtained from Isfahan University of Medical Sciences, Iran, housed in a $12 \mathrm{~h}$ lightdark cycle (lights on from 7:00 a.m. to 7:00 p.m.), temperature of $22-24^{\circ} \mathrm{C}$ with available food and water ad libitum throughout the experiment. One week after adaptation, rats were divided into the following four experimental groups: control $(n=8)$ : housed four rats per cage and received normal saline for 21 days; $\operatorname{VitC}(n=8)$ : housed four rats per cage and received VitC for 21 days; $\mathrm{CIS}(\mathrm{n}=8)$ : rats were housed individually in the cages and received normal saline for 21 days; CIS and VitC (CIS+ VitC, $n=8$ ): rats were housed individually in the cages and received VitC. Individual housing for 3 weeks was used as a model of CIS during which rats had normal auditory and olfactory signals, but had no any visual or tactile contact with other rats (Kalshetti et al. 2015; Schipper et al. 2018). Acid ascorbic solution $(500 \mathrm{mg} / \mathrm{kg} / \mathrm{lml})$ and volume-matched saline were administrated to the rats by gavage daily at 12:00 a.m. for 3 weeks.

Food intake, hormone and body weight measurement. To determine the effect of VitC on appetite and metabolism, the consumption of standard chow was assessed daily as daily food intake during 3 weeks and on days 1, 7, 14 and 21 following 18-20-h food deprivation as fasting food intake. For fasting feeding, after being gavage with VitC or saline, the fasted rats were given access to a pre-measured amount of food pellets, and $3 \mathrm{~h}$ later, the amount of food intake was evaluated by subtracting the weight of the remaining food and crumbs from the initial weight of the food. The consumed food (in grams) was divided by the number of rats housed in each cage as the amount consumed for fasting or $24 \mathrm{~h}$ consumption for each animal. VitC administration was performed on 9:0010:00 a.m. for fasting and 12:00 a.m. for daily food intake and food measurements were performed on 12:00 a.m. to 1:00 p.m.

Furthermore, body weight was measured on days $1,7,14$ and 21 of the experiment and the body weight differences (BWD = BW Day21-BW Dayl) were evaluated.

For hormone assessments, tail blood sampling technique was used to collect blood at the amount of $500 \mu \mathrm{l}$ around 8:00 a.m. on days 1, 7, 14 and 21 after 18-20-h food deprivation. The serum samples were stored at $-80^{\circ} \mathrm{C}$ until processed for biochemical assessment. Serum acyl ghrelin was measured using rat ghrelin ELISA kit with intra-assay precision $\mathrm{CV}<10 \%$ (Zellbio Company ELISA kit-Germany).

Sucrose preference test. The sucrose preference test was conducted on day 22 , as previously described (Liu et al. 2014). Initially, there were two bottles of 1 $\mathrm{m} / \mathrm{v} \%$ sucrose solution located in each cage for $48 \mathrm{~h}$ to let the animals adapt to the sucrose solution, then removed two bottles for $4 \mathrm{~h}$. The animals were then subjected to two weighted bottles for $1 \mathrm{~h}$, one filled with $1 \mathrm{~m} / \mathrm{v} \%$ sucrose solution and the other filled only with water. Sucrose preference (\%) was assessed as (sucrose consumption)/(sucrose consumption+water consumption). During the test, VitC or saline treatment was continued. 
Tissue sampling and histological staining. At the end of the experiment (on day 25), the rats were sacrificed with a guillotine under deep anesthesia via subcutaneous injection of a mixture of ketamine $(80 \mathrm{mg} / \mathrm{kg})$ and xylazine $(8 \mathrm{mg} / \mathrm{kg})$. Stomachs were rapidly removed and fixed in $10 \%$ formalin. After dehydration with an ascending ethanol series, the tissues were cleared with xylene and inserted in paraffin for 3-6 $\mathrm{h}$ and then blocked. For histological studies, a series of sections with $5 \mu \mathrm{m}$ thickness were prepared from the beginning, middle and end of the gastric tissue by a rotary microtome (Leica, Vienna, Austria). After deparaffinization, hematoxylin staining was conducted for $4 \mathrm{~min}$, followed by eosin staining for $20 \mathrm{~s}$. The sections were then dehydrated with alcohol, mounted and photographed digitally.

Statistical analysis. The results were expressed as mean \pm SEM and analyzed in SPSS 23 software. The one-way ANOVA post hoc Tukey test was run to have a one-way comparison and paired sample t-test to evaluate within group changes. For observing frequencies in alternative times, the repeated measures tests were conducted. The statistical significance in all run tests is $\mathrm{p}<0.05$.

\section{Results}

Serum ghrelin concentration. Repeated measures ANOVA was performed to determine the pattern of serum ghrelin changes over time (Figure 1). Results indicated a significant interaction among groups and time (time [repeated measures ANOVA, F( 3 , $23)=42.35 ; \mathrm{p}=0.01]$ as well as interaction intervention *time [repeated measures, $\mathrm{F}(9,23)=7.86 ; \mathrm{p}=0.01])$ (Table 1). ANOVA results indicated a significant difference between groups on days 1, 7 and 14. On first day, VitC, CIS and CIS +VitC significantly decreased ghrelin concentration compared with control ( $p=0.03$ and $p=0.01)$. As shown in Figure 1, ghrelin concentration was enhanced in VitC group compared to other groups on day 7 ( $\mathrm{p}<0.001$ vs. control, $\mathrm{p}<0.01$ vs. CIS and CIS + VitC) and on day 14 ( $\mathrm{p}<0.001$ vs. control, $\mathrm{p}<0.01$ vs. CIS, except to CIS $+\mathrm{VitC})$. Ghrelin concentration was also enhanced in CIS group compared with controls on day 7 and day 14 ( $\mathrm{p}<0.05$ vs. control). For CIS + VitC group, a significant difference in ghrelin concentration was observed compared to control group on day 14 . There was a marked difference in ghrelin concentration between VitC group and control group on day $21(p<0.05)$. The results of time effect in each group showed that ghrelin concentration was increased in VitC, CIS and CIS+VitC groups over the time $(\mathrm{p}<0.001$ for VitC group, for CIS and CIS + VitC groups $\mathrm{p}<0.01)$. Although the time effect indicated significant changes up to day 21 only for $\mathrm{VitC}$ group.

Feeding behavior. The consumption of standard chow was assessed on 1, 7, 14 and 21 days after 18-20-h food deprivation as fasting food intake and $24 \mathrm{~h}$ cumulative food intake as daily food intake during 21 days.

The results from two way ANOVA statistical analysis of fasting food intake indicated a signifi-

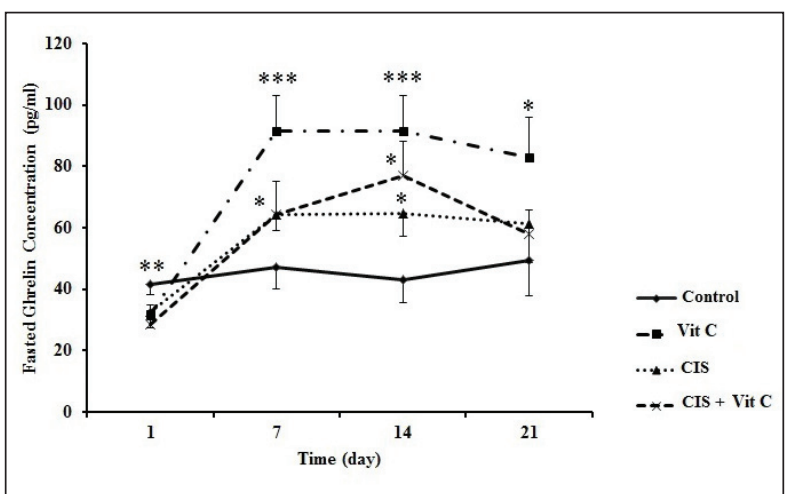

Figure 1. The pattern of serum ghrelin concentration changes (pg/ml) over time (21 days). Data are shown as mean \pm SEM; ${ }^{*} \mathrm{p}<0.05 ;{ }^{*} \mathrm{p}<0.01 ;{ }^{* *} \mathrm{p}<0.001$ vs. Control. Abbreviations: VitC - vitamin C; CIS - chronic social isolation stress.

Table 1

Analysis of repeated ghrelin level $(\mathrm{pg} / \mathrm{ml})$ measurements at different time points

\begin{tabular}{lccccccc}
\hline Group & Day 1 & Day 7 & Day 14 & Day 21 & $\begin{array}{c}\text { P1 } \\
\text { (Time) }\end{array}$ & $\begin{array}{c}\text { P2 } \\
\text { (Time*Intervention) }\end{array}$ & $\begin{array}{c}\text { P3 } \\
\text { (Intervention) }\end{array}$ \\
\hline Control & $41.70 \pm 10.22$ & $47.28 \pm 7.20$ & $43.03 \pm 7.61$ & $49.18 \pm 11.25$ & 0.16 & & \\
VitC & $31.66 \pm 3.26$ & $91.38 \pm 11.80$ & $91.38 \pm 7.80$ & $82.85 \pm 13.11$ & 0.001 & 0.01 & 0.004 \\
CIS & $32.46 \pm 5.01$ & $64.32 \pm 5.10$ & $64.22 \pm 8.51$ & $61.12 \pm 12.36$ & 0.01 & & \\
CIS+VitC & $28.30 \pm 1.55$ & $64.43 \pm 10.82$ & $76.90 \pm 11.20$ & $57.88 \pm 8.05$ & 0.01 & & \\
P4 & 0.01 & $<0.001$ & $<0.001$ & 0.05 & & \\
\hline
\end{tabular}

Data are presented as mean \pm SEM. P1, P2, P3 - at the 5\% level of repeated measures test; P4 - at the 5\% level of ANOVA test. Abbreviations: VitC - vitamin C; CIS - chronic social isolation. 
cant difference among four studied groups over time [interaction: $\mathrm{F}(9,28)=3.25 ; \mathrm{p}=0.0018$, time: $\mathrm{F}(3,28)=6.87 ; \mathrm{p}=0.0003$ and groups: $\mathrm{F}(3,28)=20.77$; $\mathrm{p}<0.0001$, Figure 2A]. On day 1, a significant reduction in fasting food intake was observed between control and VitC groups $(\mathrm{p}<0.05)$ and this reduction was abolished on days 7, 14 and 21. No difference was found among four studied groups on day 7. Isolated rats exhibited significant increases in fasting food intake after $18-20$-h food deprivation compared to control group on days 14 and 21 ( $\mathrm{p}<0.05$ and $\mathrm{p}<0.01$, respectively). There was a significant difference in food intake among CIS+VitC and control groups only on day $14(\mathrm{p}<0.01)$. Importantly, on day 21 , a significant reduction of fasting food intake was observed in $\mathrm{CIS}+\mathrm{VitC}$ and VitC groups compared to CIS group $(\mathrm{p}<0.01$ and $\mathrm{p}<0.001$, respectively).

Figure $2 \mathrm{~B}$ presents the effects of regular consumption of VitC/CIS on 24-h cumulative food intake over time. Two-way ANOVA analysis indicated a signifi-

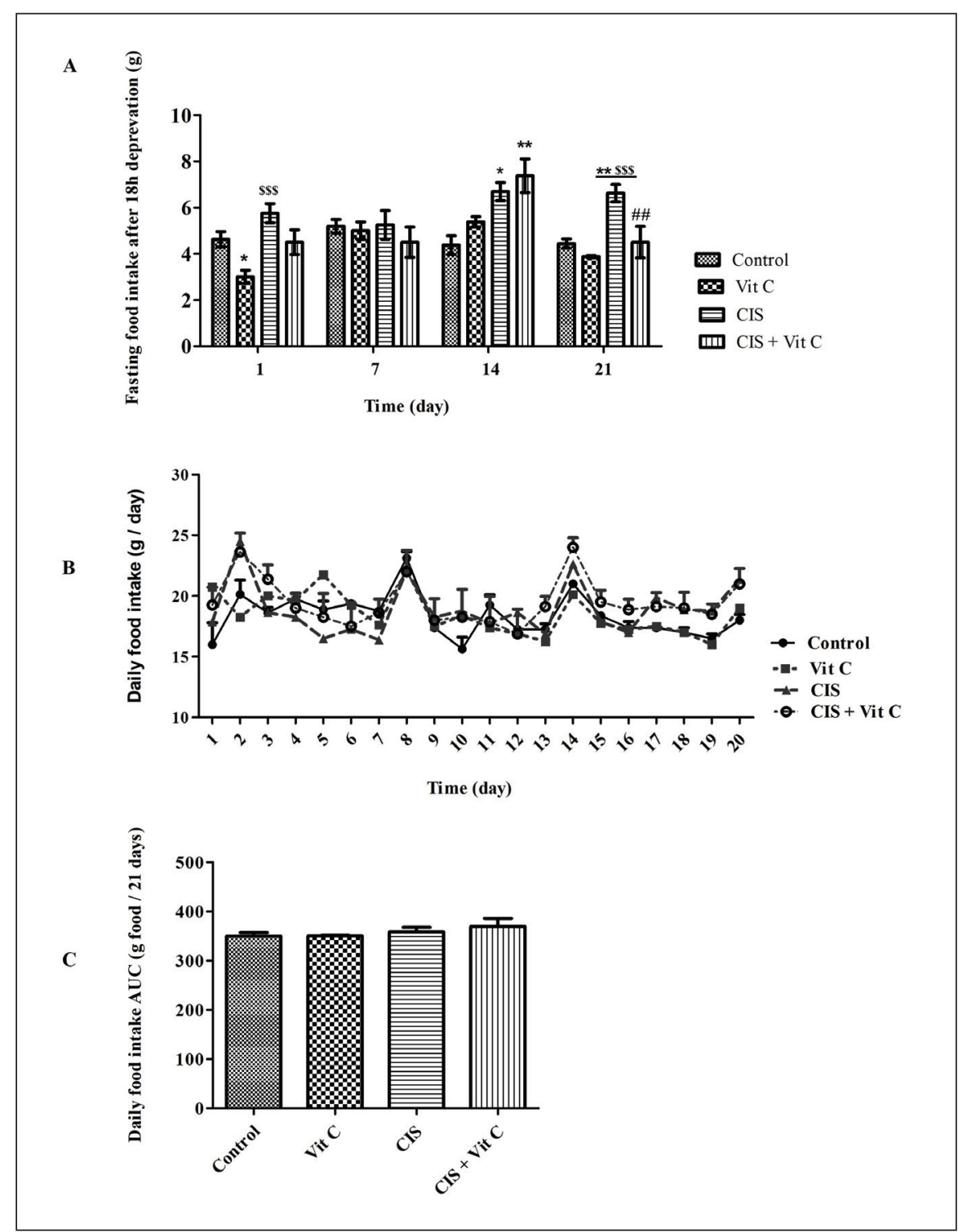

Figure 2. Effects of vitamin $\mathrm{C}(\mathrm{VitC})$ and chronic isolation stress (CIS) on fasting (g/3 h) and daily food intake (g/day). A) Average fasting food intake on days 1, 7, 14 and 21 after 18-20-h food deprivation; B) the pattern of daily food intake over time (3 weeks); C) total area under curve (AUC) of daily food intake for control and experimental groups during 3 weeks. Data are shown as mean $\pm \operatorname{SEM}(\mathrm{n}=8) ;{ }^{*} \mathrm{p}<0.05 ;{ }^{* *} \mathrm{p}<0.01$ vs. Control; $\$$ compared to vitamin C; \# compared to CIS. 


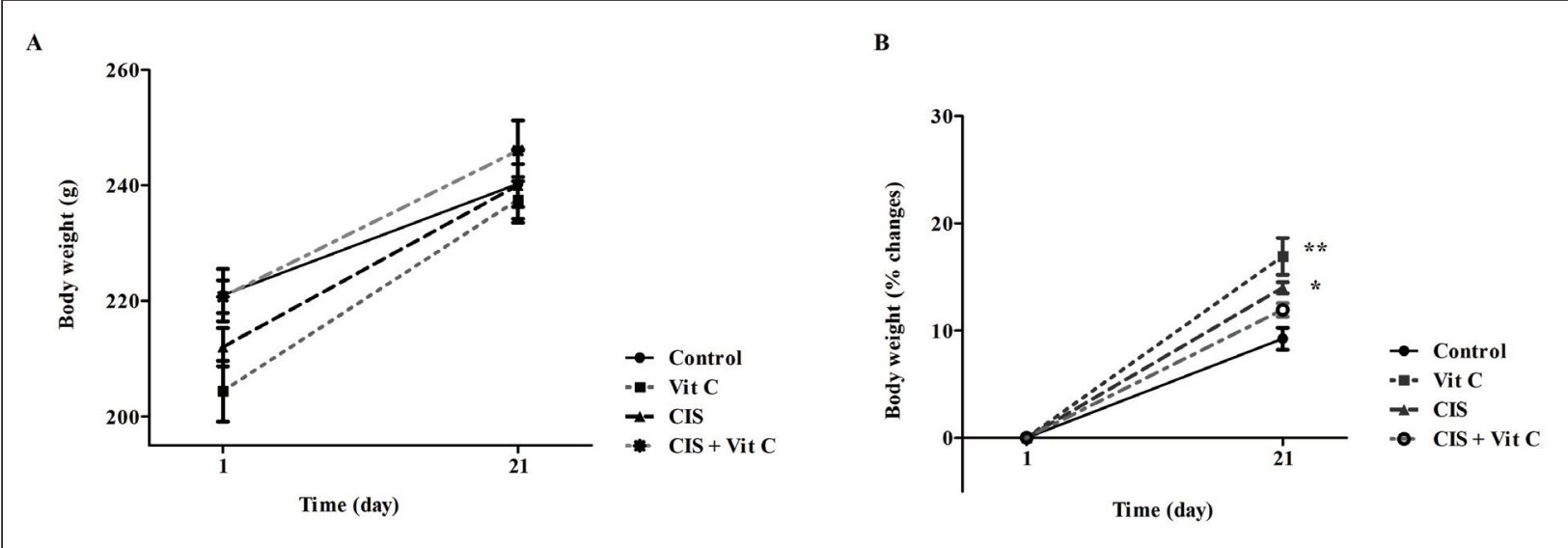

Figure 3. Effects of vitamin C (VitC) and chronic isolation stress (CIS) on body weight gain. A) The trend of body weight gain for control and experimental groups between first and 3 weeks; B) percentage of the body weight change for each group. Data are shown as mean $\pm \mathrm{SEM} ;{ }^{*} \mathrm{p}<0.05 ;{ }^{* *} \mathrm{p}<0.01$ vs. Control.

cant interaction $[\mathrm{F}(3,57)=2.98 ; \mathrm{p}<0.000]$, however, no significant differences were found among groups $[\mathrm{F}(3,28)=0.96 ; 0.4246]$. Further analysis was performed by the data from time points to calculate the area under curve (AUC; g/day) for daily food intake of each group. Total AUC of daily food intake for control and other experimental groups during 3 weeks did not indicate no significant difference between groups ( $p=0.48$, Figure $2 \mathrm{C}$ ).

Body weight changes. Figure $3 \mathrm{~A}$ shows the main effects of VitC and CIS on body weight changes. Repeated measures were performed to determine the effect of stress/VitC on body weight over time. In this regard, body weight for first and last days (1 and 21days) were considered for analysis. We found that there is no significant interaction of intervention ${ }^{\star}$ time $[F(3,28)=1.97, p=0.14]$, (Figure 3A). Because the baseline weight of the animals varied among groups, two-way ANOVA was performed for the calculation of the percentage of changes in body weight. Results showed a significant interaction of intervention ${ }^{\star}$ time $[F(3,28)=9.04 ; p=0.0002]$. The percentage of changes in body weight in VitC and CIS groups was significantly greater than control group, whereas, no significant difference was seen between CIS + VitC and control groups.

Sucrose intake. We ascertained whether VitC in context of CIS could affect sucrose intake. Sucrose intake is a behavioral test for the assessment of isolation stress-induced depressive-like behavioral phenotype. The sucrose preference percentage of isolated rats was markedly lower $(\mathrm{p}=0.006)$ than that of controls (Figure 4). However, sucrose preference in isolated rats treated with VitC did not differ from the

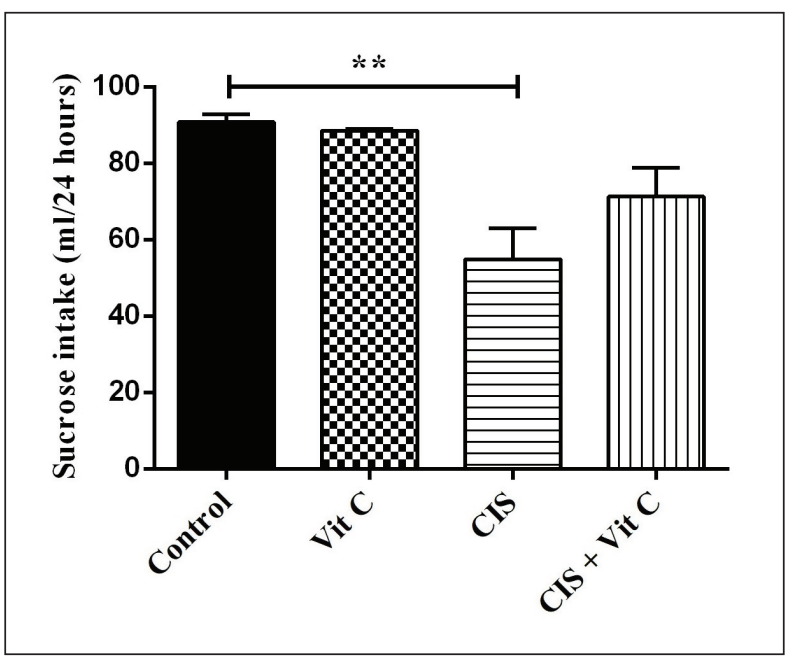

Figure 4. Effects of vitamin $\mathrm{C}(\mathrm{VitC})$ and chronic isolation stress (CIS) on depressive-like behavior. Data are shown as mean \pm SEM $(n=8) ;{ }^{* *} \mathrm{p}<0.01$ vs. Control.

control. This suggests that VitC could improve the anhedonia in aloneness situation.

Histological study. The effects of treatment with $\mathrm{VitC}$ on gastric tissue were evaluated macroscopically in control and isolation stress groups (Figure 5). Histological results indicated an intact epithelial lining and clear glandular cavity in the control gastric mucosa (Figures 5A, E). Among the experimental groups, the histological lesions in isolation stress were the worst (Figures 5C, G). In stressed rats, marked histological changes were observed. Some secretory glands were fragmented and shorten in high and many holes were found in the glandular 
cavities (Figures 5C, G). Even mucosal desquamation was found in some gastric tissues from stressed rats. Treatment with VitC alleviated the pathological changes (Figures 5D, H) compared to isolated stress. Interestingly, the gastric histology status in the VitC group was even better than that in the control group (Figures 5B, F). The higher mucosa layer was found, with higher cell density and apparently more active in the VitC-treated group.

\section{Discussion}

In the present study, we examined the impact of an antioxidant agent, VitC, on the ghrelin secretion and its orexigenic effect following CIS in adult male rats. Results indicated that the fasting plasma acylated-ghrelin concentrations and fasting food intake were significantly higher in stressed rats than those in controls, without significant weight gaining. In control rats, VitC treatment produced a marked increase in the fasting plasma ghrelin levels with weight gain that was higher in this group than that in stressed and non-treated control rats, while food intake was kept at the normal levels. The combination of isolation stress and VitC in isolated rats treated by VitC, however, did not induce significant synergistic effects on ghrelin level and ghrelin concentration was kept in this group near that in isolation stress.

There are three main neuroendocrine systems mediating most elements of the stress response, the HPA axis to release glucocorticoids, the sympatho- adrenomedullary axis triggering the release of epinephrine and norepinephrine and finally, several peptides recently identified such as ghrelin (Abizaid 2019). Ghrelin has been shown to enhance hypothalamic neuropeptide Y and AGRP mRNA levels and orexin genes in several chronic stress models in rodents (Jeong et al. 2013; Yamada et al. 2015). Chronic stress is also associated with an enhancement in plasma acyl-ghrelin (Asakawa et al. 2001; Lutter et al. 2008a; Kumar et al. 2013; Patterson et al. 2013), which persists beyond the cessation of prolonged stressor exposure (Yousufzai et al. 2018). Our data also showed a sharp ghrelin elevation in chronic isolated rats with a peak on day 7 that persisted up to end of experiment on day 21.

While numerous studies have indicated the therapeutic impacts of vitamins in certain kinds of cancer, inflammation, and cardiovascular diseases, few researches have explored a role of vitamins in modulation of ghrelin secretion as a component of the stress response. Daily intake of vitamin D, as an antioxidant, has been shown to enhance plasma ghrelin concentrations (Hajimohammadi et al. 2017). Moreover, treating the diabetic rats with curcumin, which has antioxidant activity, results in a marked increase in the content of ghrelin in the stomach and plasma ( $\mathrm{Xu}$ et al. 2013). Hence, it is tempting to speculate that antioxidants could affect ghrelin secretion. As expected, we observed a strong secretory release of ghrelin in response to $\mathrm{VitC}$ in control group over time. However, VitC-induced increase in

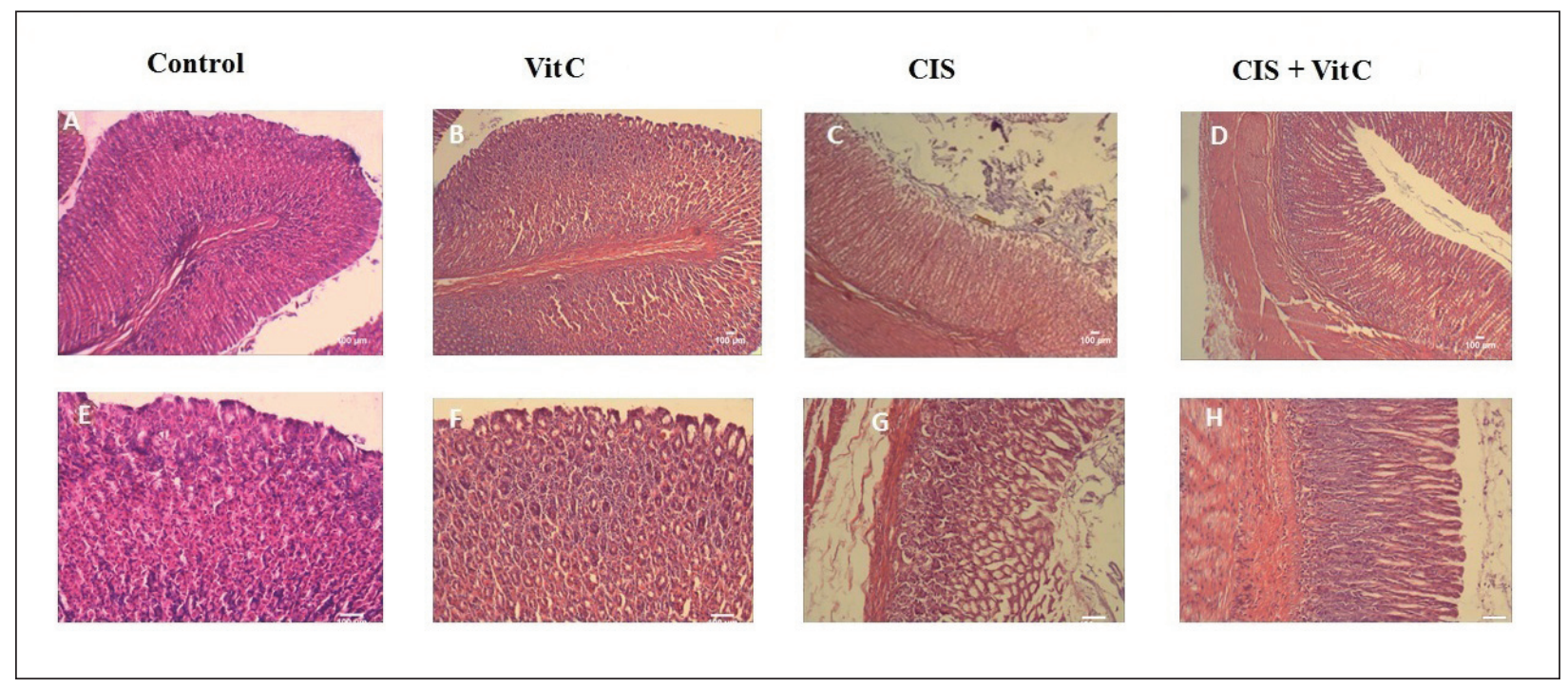

Figure 5. Histological representation of gastric tissue, mucosa layer, following vitamin $\mathrm{C}$ treatment in chronic isolated rats after hematoxylin and eosin staining. Magnification for A, B, C and D: X=40; magnification for E, F, G and H: X=100. Abbreviations: VitC - Vitamin C; CIS - chronic social isolation stress. 
ghrelin levels may be partly independent of its antioxidant activity. There are some evidences indicating that an enhancement in plasma ghrelin level is regulated by sympathetic nerves innervating ghrelinsecreting cells and/or by circulating epinephrine and norepinephrine that can influence ghrelin cells via an effect on adrenergic receptors (Zhao et al. 2010; Gupta et al. 2019). On other hand, VitC has been found to enhance $\beta$-adrenergic activity and increase potency and duration of beta-adrenergic agonists' effects in smooth muscle. Our results also revealed that $\mathrm{VitC}$ protects the gastric fundus mucosa against chronic stress induced-injury and even it enhances glandular cells in control group compared to nontreated control group, suggesting that a significant increase in ghrelin levels in VitC group, at least in part, may be due to a positive direct effect of VitC on glandular cells in gastric tissue. Thus, the extent of ghrelin secretion in VitC-treated rats may be regulated through antioxidant and adrenergic pathways and via inducing gastric mucosal cell proliferation. In stressed rats treated with $\mathrm{VitC}$, the amount of ghrelin secreted was as much as non-treated stressed rats, suggesting that $\mathrm{VitC}$ is likely to be employed in neutralization of oxidative stress than other pathways (not related to ghrelin secretion) under stress condition.

Stress-induced changes in the ghrelin system have been shown to impact eating and metabolism. For instance, rodents exposed to chronic social stress (Moles et al. 2006; Lutter et al. 2008b) or mild restraining exhibit hyperphagia and preference for food rewards, whereas chronic social stress-exposed mice lacking GHSR do not so (Yanagi et al. 2018). Similarly, wild-type mice receiving long term GHSR antagonist infusion following chronic social stress exhibited a reduction in stress-induced weight gain and hyperphagia (Chuang 2011). Yamada et al. (2015) have also observed that food intake was significantly increased in sub-chronic isolated young mice, whereas food intake was suppressed during the first several hours of isolation. In the present study, we found an increase in post-fasting food intake on days 14 and 21 of CIS induction, without any change in 24-h cumulative or daily food intake compared with control group. In our study, a marked increase in the ghrelin levels from day 7 onward in the VitC group was not associated with a marked change in fasting and daily food intake. Also, there were no marked changes in weight gain among the experimental groups. In agreement with other studies, our data indicate that CIS results in an enhancement in fasting ghrelin and fasting food intake in stressed rats, which may be an underlying mechanism of hyperphagia and obesity under stress condition. Although, we have no direct evidence to explain the inconsistency between increased ghrelin without enhanced appetite in chronic VitC-treated rats, a possible explanation may be due to an effect of $\mathrm{VitC}$ on responsiveness of GHSR to ghrelin. This is because Chebani et al. (2016), using an animal model carrying a GHSR mutation that preserves GHSR cell surface abundance, but selectively alters GHSR signaling, found that rats with GHSR mutation exhibited increased responses to submaximal doses of ghrelin and had a more stable body weight under caloric restriction, a state that enhances endogenous ghrelin level, while under standard housing states, GHSR mutated rats exhibited enhanced body weight and adiposity without increased appetite (Chebani et al. 2016). This seems to be a favorable condition in which VitC can maintain its antioxidant role for ghrelin secretion under stress condition, while not contributing to increase appetite.

In addition to food intake, ghrelin also plays a principal role in mood, and potentially increases resilience to stress (Huang et al. 2017). While there was a marked enhancement in plasma ghrelin levels in isolated group, the animals displayed a significant depression-like behavior and VitC could produce a significant antidepressant-like effect in this group. Previous studies have suggested that ghrelin help to cope with stress and causes neuroprotective effects against brain injury in chronic stress. Studies have also revealed neuroprotective and neuromodulatory roles of $\mathrm{VitC}$ as an anxiolytic, antidepressant and antiepileptic drug (Kocot et al. 2017). It has also been proposed that the therapeutic potential impacts of high levels of dietary acid ascorbic in mental illnesses such as anxiety and depression may depend on protecting the 5-HT molecule against oxidation (Ballaz and Rebec 2019). Hence the alleviation of depression-like behaviors may in part be due to an interaction of VitC with ghrelin signaling through an effect on monoamine systems. Sympathetic receptors appear to be more responsive with $\mathrm{VitC}$ treatment under stress conditions, hence, $\mathrm{VitC}$ in isolated rats, which have an elevated ghrelin, may provide a good antidepressant, whereas the same level of endogenous ghrelin is also seen under stress conditions. Although another possibility is that antioxidant, but not just ghrelin, effects of VitC may also facilitate to reverse the depression-like behaviors.

Together, our data indicate, as the first evidence, that chronic VitC supplementation could increase endogenous ghrelin concentration and suggests that 
VitC may have an improving effect against damage of CIS on eating, metabolism, and depression-like behaviors. The elevation of ghrelin under stress conditions may be a homeostatic adaptation to stress that affects the amount of food intake in fasting.

\section{Acknowledgments}

This work was supported by the student research committee (197077) of the Isfahan University of Medical Sciences, Isfahan, Iran.

\section{References}

Aasheim ET, Bohmer T. Low preoperative vitamin levels in morbidly obese patients: a role of systemic inflammation? Surg Obes Relat Dis 4, 779-780, 2008.

Abdali D, Samson SE, Grover AK. How effective are antioxidant supplements in obesity and diabetes? Med Princ Pract 24, 201-215. 2015.

Abizaid A. Stress and obesity: the ghrelin connection. J Neuroendocrinol 31, e12693, 2019.

Asakawa A, Inui A, Kaga T, Yuzuriha H, Nagata T, Fujimiya M, Katsuura G, Makino S, Fujino MA, Kasuga M. A role of ghrelin in neuroendocrine and behavioral responses to stress in mice. Neuroendocrinology 74, 143-147, 2001.

Ballaz SJ, Rebec GV. Neurobiology of vitamin C: Expanding the focus from antioxidant to endogenous neuromodulator. Pharmacol Res 146, 104321, 2019.

Beheshti F, Karimi S, Vafaee F, Shafei MN, Sadeghnia HR, Hadjzadeh MAR, Hosseini M. The effects of vitamin C on hypothyroidism-associated learning and memory impairment in juvenile rats. Metab Brain Dis 32, 703-715, 2017.

Bisbal C, Lambert K, Avignon A. Antioxidants and glucose metabolism disorders. Curr Opin Clin Nutr Metab Care $13,439-446,2010$.

Chebani Y, Marion C, Zizzari P, Chettab K, Pastor M, Korostelev M, Geny D, Epelbaum J, Tolle V, Morisset-Lopez S, Pantel J. Enhanced responsiveness of Ghsr Q343X rats to ghrelin results in enhanced adiposity without increased appetite. Sci Signal 9, ra39, 2016.

Chuang JC, Zigman JM. Ghrelin's roles in stress, mood, and anxiety regulation. Int J Pept 2010, 460549, 2010.

Chuang JC. Ghrelin mediates stress-induced food-reward behavior in mice. J Clin Invest 121, 2684-2692, 2011.

Cummings DE, Weigle DS, Frayo RS, Breen PA, Ma MK, Dellinger EP, Purnell JQ. Plasma ghrelin levels after dietinduced weight loss or gastric bypass surgery. N Engl J Med 346, 1623-1630, 2002.

Gupta D, Chuang JC, Mani BK, Shankar K, Rodriguez JA, Osborne-Lawrence S, Metzger NP, Zigman JM. $\beta 1$ adrenergic receptors mediate plasma acyl-ghrelin elevation and depressive-like behavior induced by chronic psychosocial stress. Neuropsychopharmacology 44, 1319-1327, 2019.

Hajimohammadi M, ShabBidar S, Neyestani TR. Consumption of vitamin D fortified yogurt drink increased leptin and ghrelin levels but reduced leptin to ghrelin ratio in type 2 diabetes patients: a single blind randomized controlled trial. Eur J Nutr 56, 2029-2036, 2017.

Huang HJ, Zhu XC, Han QQ, Wang YL, Yue N, Wang J, et al. Ghrelin alleviates anxiety- and depression-like behaviors induced by chronic unpredictable mild stress in rodents. Behav Brain Res 326, 33-43, 2017.

Jeong JY, Lee DH, Kang SS. Effects of chronic restraint stress on body weight, food intake, and hypothalamic gene expressions in mice. Endocrinol Metab 28, 288-296, 2013.

Kalshetti PB, Alluri R, Mohan V, Thakurdesai PA. Effects of 4-hydroxyisoleucine from Fenugreek seeds on depression-like behavior in socially isolated olfactory bulbectomized Rats. Pharmacogn Mag 11(Suppl 3), S388S396, 2015.

Kristenssson E, Sundqvist M, Astin M, Kjerling M, Mattsson H, Dornonville de la Cour C, Hakanson R, Lindstrom E. Acute psychological stress raises plasma ghrelin in the rat. Regul Pept 134, 114-117, 2006.

Kocot J, Luchowska-Kocot D, Kielczykowska M, Musik I, Kurzepa J. Does vitamin C influence neurodegenerative diseases and psychiatric disorders? Nutrients 9, 659, 2017.

Kumar J, Chuang JC, Na ES, Kuperman A, Gillman AG, Mukherjee S, Zigman JM, McClung CA, Lutter M. Differential effects of chronic social stress and fluoxetine on meal patterns in mice. Appetite 64, 81-88, 2013.

Liu D, Wang Z, Gao Z, Xie K, Zhang Q, Jiang H, Pang Q. Effects of curcumin on learning and memory deficits, BDNF, and ERK protein expression in rats exposed to chronic unpredictable stress. Behav Brain Res 271, 116-121, 2014.

Lutter M, Sakata I, Osborne-Lawrence S, Rovinsky SA, Anderson JG, Jung S, Birnbaum S, Yanagisawa M, Elmquist JK, Nestler EJ, Zigman JM. The orexigenic hormone ghrelin defends against depressive symptoms of chronic stress. Nat Neurosci 11, 752-753, 2008a. 
Lutter M, Krishnan V, Russo SJ, Jung S, McClung CA, Nestler EJ. Orexin signaling mediates the antidepressant-like effect of calorie restriction. J Neurosci 28, 3071-3075, 2008b.

Moles A, Bartolomucci A, Garbugino L, Conti R, Caprioli A, Coccurello R, Rizzi R, Ciani B, D’Amato FR. Psychosocial stress affects energy balance in mice: modulation by social status. Psychoneuroendocrinology 31 , 623-633, 2006.

Muller TD, Nogueiras R, Andermann ML, Andrews ZB, Anker SD, Argente J, et al. Ghrelin. Mol Metab 4, 437-460, 2015.

Ochi M, Tominaga K, Tanaka F, Tanigawa T, Shiba M, Watanabe T, Fujiwara Y, Oshitani N, Higuchi K, Arakawa T. Effect of chronic stress on gastric emptying and plasma ghrelin levels in rats. Life Sciences 82, 862-868, 2008.

Ortega RM, Rodriguez-Rodriguez E, Aparicio A, Jimenez-Ortega AI, Palmeros C, Perea JM, Navia B, Lopez-Sobaler AM. Young children with excess of weight show an impaired selenium status. Int J Vitam Nutr Res 82, 121-129, 2012.

Ouach V, Bloch M, Rosenberg N, Gilad S, Limor R, Stern N, Greenman Y. The acute ghrelin response to a psychological stress challenge does not predict the poststress urge to eat. Psychoneuroendocrino 32, 693-702, 2007.

Patterson ZR, Khazall R, Mackay H, Anisman H, Abizaid A. Central ghrelin signaling mediates the metabolic response of C57BL/6 male mice to chronic social defeat stress. Endocrinology 154, 1080-1091, 2013.

Pizzino G, Irrera N, Cucinotta M, Pallio G, Mannino F, Arcoraci V, Squadrito F, Altavilla D, Bitto A. Oxidative stress: harms and benefits for human health. Oxid Med Cell Longev 2017, 8416763, 2017.

Razzoli M, Sanghez V, Bartolomucci A. Chronic subordination stress induces hyperphagia and disrupts eating behavior in mice modeling binge-eating-like disorder. Front Nutr 1, 1-10, 2015.

Schipper L, Harvey L, van der Beek EM, van Dijk G. Home alone: a systematic review and meta-analysis on the effects of individual housing on body weight, food intake and visceral fat mass in rodents. Obes Rev 19, 614-637, 2018.

Strauss RS. Comparison of serum concentrations of alpha-tocopherol and beta-carotene in a cross-sectional sample of obese and nonobese children (NHANES III). National Health and Nutrition Examination Survey. J Pediatr 134, 160-165, 1999.

Teegarden SL, Bale TL. Effects of stress on dietary preference and intake are dependent on access and stress sensitivity. Phys Behav 93, 713-723, 2008.

Valdecantos MP, Perez-Matute P, Martinez JA. Obesity and oxidative stress: role of antioxidant supplementation. Rev Invest Clin 61, 127-139, 2009.

Xu L, Li Z, Guo F. Curcumin improves expression of ghrelin through attenuating oxidative stress in gastric tissues of streptozotocin-induced diabetic gastroparesis rats. Eur J Pharmacol 718, 219-225, 2013.

Yamada C, Saegusa Y, Nahata M, Sadakane C, Hattori T, Takeda H. Influence of aging and gender differences on feeding behavior and ghrelin-related factors during social isolation in mice. PLoS One 10, e0140094, 2015.

Yanagi S, Sato T, Kangawa K, Nakazato M. The homeostatic force of ghrelin. Cell Metabolism 27, 786-804, 2018.

Yousufzai M, Harmatz E, Shah M, Malik M, Goosens K. Ghrelin is a persistent biomarker for chronic stress exposure in adolescent rats and humans. Transl Psychiatry 8, 74, 2018.

Zhao TJ, Sakata I, Li RL, Liang G, Richardson JA, Brown MS, Goldstein JL, Zigman JM. Ghrelin secretion stimulated by \{beta\}1-adrenergic receptors in cultured ghrelinoma cells and in fasted mice. Proc Natl Acad Sci USA 107, $15868-15873,2010$. 